Welcher Maximalwert der wahrscheinlichere ist, sei dahingestellt; ich meine, daß 0.8 nicht zu groß ist.

Später habe ich versucht, den Schweif am 5. Mai I 759 als gebrochene Linie in Rechnung zu ziehen und unter drei verschiedenen Annahmen über eine solche Zerlegung als Länge des Schweifes 1.02, bezw. 0.8 I und 0.69 gefunden. Man kann also, wie man sich auch bemühen mag, für den 5. Mai eine wahre Schweiflänge von nur mäßiger Größe zu erhalten, nur schwer unter 0.7 oder 0.8 herunterkommen.

Vergleicht man die in der zitierten Zusammenstellung (A. N. 4404) enthaltenen Schweiflängen und faßt dabei sowohl die Mittelwerte $C_{1}$ als auch die Maximalwerte $C_{2}$ ins Auge, so sieht man zunächst, daß die größten Längen in der Erscheinung von $145^{6}$ beobachtet worden sind, in welcher die Erdnähe mit der Sonnennähe zusammenfiel. (Juni-Er-

Wien, I9 Io Mai 20. scheinung). Wesentlich kleiner sind die der Jahre I 53 I und I682 (August-September-Erscheinungen), und am kleinsten die der Jahre 1607 und 1835 , in denen der Komet schon einen Monat vor dem Perihel in die Erdnähe gekommen und also bei erst zunehmender Schweifentwickelung beobachtet worden ist. (Herbst-Erscheinungen). In der Erscheinung von 1759 , in welcher er, so wie in der von I9 Io, erst nach dem Perihel in die Erdnähe gekommen ist (Frühjahrs-Erscheinungen), ist blob der Maximalwert besonders groß, nicht der Mittelwert; dies findet seine Erklärung darin, daß die Erdnähe einerseits mit der auch nach dem Perihel noch einige Zeit fortdauernden bedeutenden Schweifentwickelung und andererseits ziemlich nahe mit dem Durchgang der Erde durch die Ebene der Kometenbahn $(L=\delta)$ zusammentraf.

\title{
Fortgesetzte Beobachtungen und Mitteilungen vom Halleyschen Kometen.
}

\section{F. Holetschek.}

[Mit einer Tafel].

Observations at 'Transvaal Observatory. 9-inch Refractor.

\begin{tabular}{|c|c|c|c|c|c|c|c|c|c|c|c|c|c|c|}
\hline 1910 & Gr. M. T. & $\Delta \alpha$ & $\not d \delta$ & Cp. & Obs. & & $\alpha$ app. & $\lg p \cdot d$ & $\delta$ ap & & $\lg p \cdot A$ & Red. ad & 1. app. & \\
\hline & $15^{\mathrm{h}} 19^{\mathrm{m}} 30^{\mathrm{s}}$ & $-I^{m} 16: 86$ & $-7^{\prime} \quad 2 l^{\prime \prime} 1$ & 3,3 & I & 23 & $5^{6^{m}} 35^{s} \cdot 55$ & $.72 \mathrm{I}_{\mathrm{n}}$ & $-\vdash 7^{\circ} 5 \mathrm{I}$ & $7 " 5$ & $0.60 I_{12}$ & $-\mathrm{I}: 30$ & -9.2 & \\
\hline I 3 & $r_{5} \quad 29$ I 3 & $\begin{array}{ll}\text { - I } & 15.97\end{array}$ & -7 I.I & 3,3 & W & 23 & $56 \quad 36.44$ & $9.720_{n}$ & $+75 \mathrm{I}$ & & $0.609 n$ & -1.30 & -9.2 & \\
\hline I 5 & 15.837 & -0 59.95 & Beyond & $4^{,-}$ & I & 23 & 5454.18 & $9.7 \times 9 n$ & +750 & \pm est. & $0.603 n$ & -1.26 & -9.1 & \\
\hline I 5 & I $5 \quad 24 \quad 8$ & - $0 \quad 59.74$ & range of & $5,-$ & W & 23 & $54 \quad 54.39$ & $9.7 \times 4 n$ & $»$ & & $0.6 \mathrm{Ion}_{\mathrm{n}}$ & -1.26 & -9.1 & \\
\hline I 5 & I $5 \quad 33 \quad 37$ & - I I.IO & screw & $2,-$ & I & 23 & $54 \quad 53$ & $9.7 \mathrm{IIn}$ & $"$ & & & $-r .26$ & -9.1 & \\
\hline I 6 & I 5 I 36 & to 58.90 & -539.2 & 5,5 & I & 23 & 544 & $9.720 \mathrm{n}$ & +748 & & $0.602 \mathrm{n}$ & $-\mathrm{r} .2 \mathrm{I}$ & -9.1 & \\
\hline I 6 & I 5 I 47 & +o $5^{8.83}$ & $-537 \cdot 7$ & 4,4 & W & 23 & $54 \quad 4.73$ & $9.7 \pm 6 n$ & +748 & 3.6 & & $--\mathrm{I} .2 \mathrm{I}$ & -9.1 & \\
\hline 18 & I 44958 & + I $39.8 \mathrm{I}$ & +414.5 & 4,4 & I & 23 & 5240.05 & $9.72 x_{n}$ & +747 & & & 一 І. I 8 & -9.0 & \\
\hline I 8 & I 5 I 44 & -0 23.35 & $-7 \times 0.3$ & 5,5 & W & 23 & $5242.6 \mathrm{I}$ & $9.717 \mathrm{n}$ & +747 & $2 I .1$ & $0.606_{n}$ & - I. $\times 5$ & -9.0 & \\
\hline 19 & r $4 \quad 48 \quad 55$ & + I 3.98 & +342.5 & 5,5 & $I$ & 23 & 524 & $9.7+9 \mathrm{n}$ & +746 & & & -- I. I 5 & -8.9 & \\
\hline I 9 & I $5 \quad O \quad 2$ & +I 3.87 & +343 & 5,5 & W & 23 & $5^{2}$ & $9.7 \times 6_{n}$ & +746 & & $0.608 n$ & - I.I 5 & -8.9 & \\
\hline 21 & 223 & to 3.82 & +323.4 & I 0,6 & I & 23 & $4 \cdot 12$ & $9.7 \times 10_{n}$ & +746 & 35.5 & $3 n$ & - I.I 2 & -8.8 & \\
\hline $2 \mathrm{I}$ & I $5 \quad$ I $5 \quad 42$ & to 40.43 & -639.5 & 5,7 & W & 23 & $5 \mathrm{I} \quad 3.24$ & $9.7 \circ 3 \mathrm{n}$ & +746 & 30.9 & $0.620_{n}$ & - I.12 & -8.8 & \\
\hline 22 & $\begin{array}{lll} & 5 & 9\end{array}$ & $\begin{array}{ll}-0 & 17.28\end{array}$ & +339.9 & 10,10 & $\mathrm{I}$ & 23 & $50 \quad 43.03$ & & +746 & 52.1 & $0.6 \mathrm{r} 8 \mathrm{n}$ & - I.I I & -8.7 & \\
\hline 22 & I5 52522 & to 19.23 & -623.0 & 10,9 & W & 23 & $5 \circ \quad 42.05$ & $9.693 \mathrm{n}$ & +746 & 47.5 & $0.626 n$ & - I.I I & -8.7 & \\
\hline 23 & I $5 \quad 1237$ & +o $4.3^{8}$ & -539.5 & 8,6 & I & 23 & $50 \quad 27.22$ & $9.699 \mathrm{n}$ & +747 & 31.0 & $0.622 n$ & -1.09 & -8.7 & \\
\hline 23 & I $5 \quad 24 \quad 59$ & $-0 \quad 3^{2.39}$ & +424.2 & I O, IO & W & 23 & $50 \quad 27.94$ & $9.689 \mathrm{n}$ & +747 & $3^{6.4}$ & $0.628 n$ & -1.09 & -8.7 & \\
\hline 24 & $\begin{array}{lll} & 5 & 9\end{array}$ & $\begin{array}{ll}-\circ & 3.48\end{array}$ & -432.4 & I 0,6 & $I$ & 23 & 5019 & & +748 & 38.2 & $0.622 n$ & $-\mathbf{I}$ & -8.6 & \\
\hline 24 & I $522 \quad 58$ & -040.29 & $+53^{2.5}$ & ro, 10 & $W$ & 23 & $50 \quad 20.05$ & $9.688_{n}$ & +748 & 44.8 & $0.629 n$ & -1.08 & -8.6 & \\
\hline
\end{tabular}

The observations are corrected for refraction. Observers: $\mathrm{I}=R . T$. A. Innes, $\mathrm{W}=W . M$. Worssell.

Mean places of the comparison stars.

\begin{tabular}{|c|c|c|c|c|c|c|c|}
\hline * & $\alpha \quad 1910.0$ & $\delta 1910.0$ & Authority & * & $\alpha 1910.0$ & $\delta$ i910.0 & Authority \\
\hline I & $23^{\mathrm{h}} 57^{\mathrm{m}} 53^{\mathrm{s}} \cdot 7 \mathrm{x}$ & $+7^{\circ} 59^{\prime} \quad 8^{\prime \prime} 8$ & $32<$ Pisc., Astr. Papers VIII. 3 & 4 & $23^{\mathrm{h}} 5^{\mathrm{I}^{\mathrm{m}}} \quad \mathrm{I}^{\mathrm{s}} \cdot 42$ & $+7^{\circ} 43^{\prime} 20^{\prime \prime} 9$ & 63 B. Pisc., As \\
\hline & $23 \quad 55 \quad 55 \cdot 39$ & $\begin{array}{lll}+7 & 5^{8} & 3 \cdot 3\end{array}$ & AG Lpz II I I 848 & 5 & $23 \quad 50 \quad 23.93$ & +753 19.2 & AG Lpz II I I 8 I 3 \\
\hline & 2353 & $+7 \quad 54 \quad 40.4$ & $A G L p z ~ I I$ I $183^{\circ}+$ Toul & & & & \\
\hline
\end{tabular}

Transvaal Observatory, I 910 Aprit 25.

R. T. A. Innes.

Photographs of Halley's Comet have been obtained at the Transvaal Observatory on the following dates:

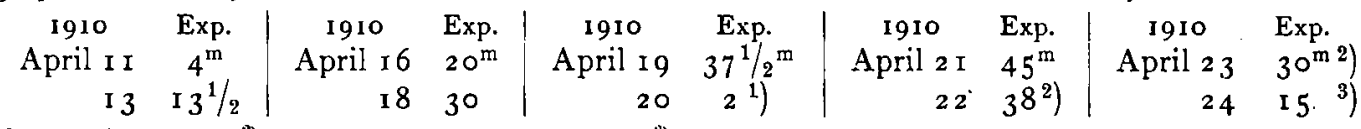

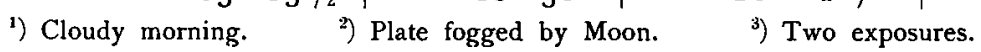




\section{Komet Halley.}

Fig. 1. Photograph by H. E. Wood, Johannesburg. 1910 April 21.

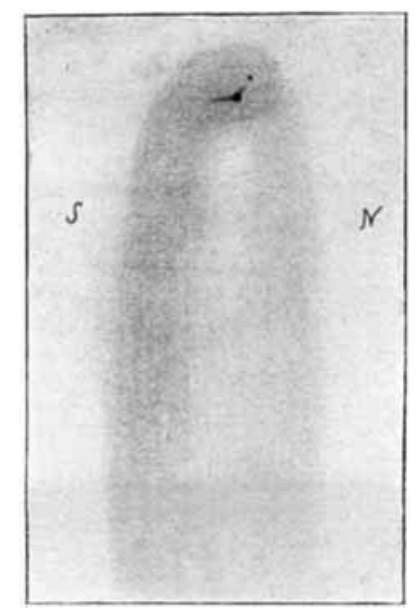

Fig. 2. Sketch of Head by R. T. H. Innes. 1910 April 24.

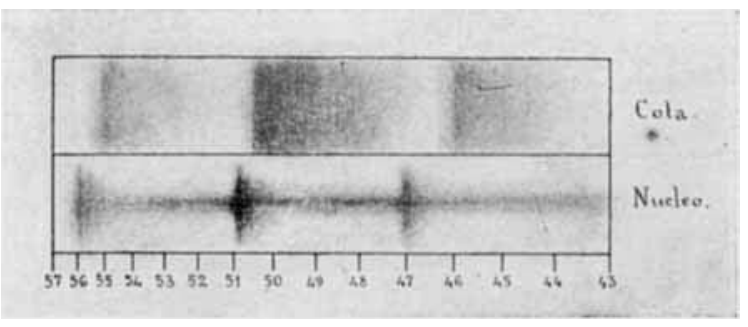

Fig. 4. F. Iñiguez. Espectro del Cometa de Halley. 1910 Mavo 9v 11.

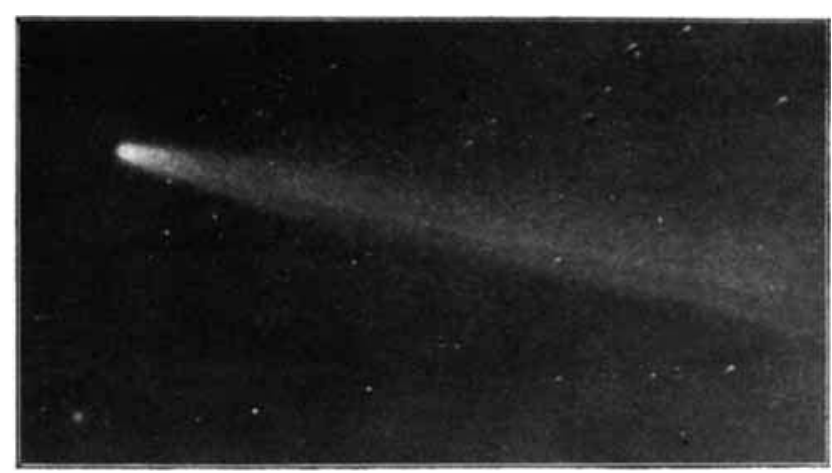

Fig. 3. F. Iñiguez. Fotograíra de la cola. 1910 Mayo 5.

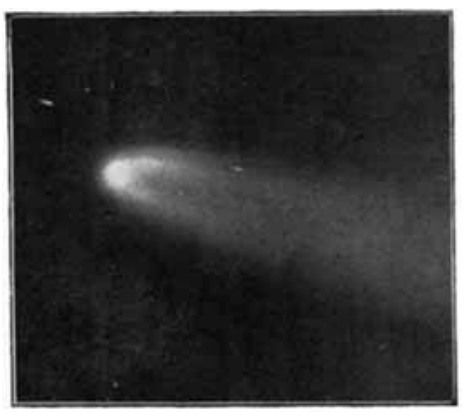

Fig. 5. F. Iñiguez. Fotografía del núcleo. 1910 Mayo 11. 
The best photograph was obtained on April 2 I (astr. date) with an exposure of 45 minutes from $4^{\mathrm{h}} 42^{\mathrm{m}}-5^{\mathrm{h}} 27^{\mathrm{m}}$ a. $\mathrm{m}$. Standard Time of $30^{\circ} \mathrm{E}$ April 22 (civil) (see Fig. I on table). The Franklin-Adams' Star Camera with a Cooke 10 inch lens of 45 inches focal length was used with Barnet Rocket Plates I 5 inches by 15 inches. The camera was guided by means of a 6 -inch finder using a 6.8 star in the comet's tail about $I^{1} / 2^{\circ}$ from the nucleus as a guiding star. The expostre was commenced shortly after the time of rising of the comet, hence the trails of stars at some distance from the comet's nucleus.

The chief characteristic of all the photographs obtained is the number of rays or streamers forming the tail of the comet. There appear to be two groups each containing two parallel wavy streamers on either side of the axis of the tail. In addition there are several side streamers showing kinks and irregularities. In several ways there is a resemblance to the tail of Morehouse's Comet r $908 \mathrm{c}$. On the original negative the length of the tail just exceeds 8 degrees. The scale of the photograph is $\mathrm{I}^{\circ}=25 \mathrm{~mm}$.

Transvaal Observatory, I 9 Io April 25. H. E. Wood.

Visual observations with a 9 -inch refractor (Magn: about I 50).

igio

April I 3. An even round nucleus.

” 16 . There is a secondary nucleus at $48^{\circ}, 12^{\prime \prime} *$ ).

" I 8. Nucleus elongated $3: 2$ in $113^{\circ}, 3^{\prime \prime} 4$ by 2.0 estimated. There is as secondary nucleus at I $54^{\circ}, 5^{\prime \prime}$. The south branch of the tail is the brighter.

» 19. No secondary nucleus seen. Nucleus and coma equal to $3^{\text {rd }}$ magn. Visible tail $2 \frac{1}{4}$ and is bright over $1 / 2^{\circ}$.

" $2 x$. Secondary nucleus seen $50^{\circ}, 12 "$, but it is indistinct. A dark space is immediately N. f. the main nucleus.

》22. Secondary nucleus $160^{\circ}$ and close to primary, like a big ill-defined double star. South branch of tail much the brighter.

»23. No secondary nucleus visible. The outpouring is concentrated on the south side.

"24. Secondary nucleus $80^{\circ}, 7^{\prime \prime}$. Both nuclei more stellar.

These observations were made at about $15^{\mathrm{h}}$ Greenwich Time. The secondary nucleus was never easily seen in a 4 -inch refractor. If this nucleus has been observed elsewhere, it is hoped that its period of revolution may be deternined and thereby a reliable estimate of the Comet's mass obtained. Our visual observations always show that the outpouring of matter is on the south side, giving the Comet quite a peculiar shape as will be shown by the sketch (Fig. 2 on table).

Transvaal Observatory, Johannesburg, i 9 io April 25.

R. T. A. Innes.

En el Observatorio de Madrid hemos podido hacer observaciones de posición y fotográficas del cometa de Halley en condiciones de cielo bastante aceptable en general y buenas en muchas ocasiones. Las posiciones observadas se publicarán tan pronto como estén reducidas. Las fotografias han sido obtenidas con un doublet de 6 pulgadas y con un objetivo fotográfico de 8 pulgadas, ambos de Grubb, colocados sobre la misma montura ecuatorial: el primero da buenas fotografías de la cola; el segundo del núcleo y partes inmediatas.

El día $\mathrm{I}^{\circ}$ de marzo es la primera vez que en la fotografía se acusa una cola muy fina y de pocos minutos de extension y se conserva así hasta el 8 de marzo, en que dejamos de ver el cometa por su proximidad al Sol.

Desde el 20 de abril podemos observar de nuevo: la cola se presenta formada de dos ramas intensas y cortas, formando ángulo muy abierto. En los dias sucesivos este ángulo va cerrándose y la cola se prolonga con bastante rapidez; el dia 25 de abril tiene una amplitud de más de $2^{\circ}$ : el dia 28 pasa de cuatro grados. Desde primero de mayo la cola pasa de ocho grados y se sale ya del campo abrazado por nuestras placas. Es notable el rápido desarrollo que ha tomado en los dias siguientes. El dia 8 de mayo pasaba de 20 grados de amplitud: el dia i i tenía más de $30^{\circ}$ : en fín, el dia I 4 Hegaba desde cerca de $\eta$ Piscium hasta más allá de $\varepsilon$ Pegasi, cerca de $60^{\circ}$.

La cola es siempre de una constitución filamentosa complicada, y sin curvatura bien acusada: únicamente el dia I 4 de mayo se insinua una inflexion del extremo hacia el Sur. Los detalles más notables que ha presentado han sido en mayo 5 (véase la lámina, Fig. 3) una ráfaga intensa y fina que se destaca en medio de la cola y que tiene una inflexión brusca á unos $5^{\circ}$ de distancia del núcleo: y el to de mayo, una ráfaga recta y débil, que se separa del resto de la cola y se extiende en linea recta por el lado Norte.

El núcleo ha presentado una condensación central cada vez más intensa en los dias sucesivos de mayo, hallándose rodeado por la cabellera, poco extensa en general: desde el io de mayo la condensación central no se acusa, confundiéndosa en un aspecto nebuloso el núcleo y la cabellera (véase la lámina, Fig. 5). Su brillo el dia I 4 era superior al de las estrellas de $I^{2}$ magnitud: el dia 13 lo pudimos observar á simple vista hasta 15 minutos antes de la salida del Sol. Un temporal de lluvia impide observar desde el 14.

En los dias 7 y 8 de mayo obtuvimos fotografías del espectro con un prisma objetivo de $20^{\circ}$ de ángulo refringente, que cubre toda la abertura del objetivo de ocho pulgadas delante del cual se coloca. I as fotografias obtenidas acusan un espectro continuo rectilineo del núcleo y tres imágenes monocromáticas de la parte central del cometa. Observado directamente el espectro con un espectroscopio de rendija colocado en la ecuatorial de Grubb de 8 pulgadas, el resultado ha sido la observación, además del espectro continuo de tres bandas brillantes, una en el amarillo, otra en el verde y otra en el azul. Las tres bandas se presentan lo mismo en el núcleo que en la cola, pero con intensidad mayor en el núcleo, y con limites diferentes. Todas las bandas se difunden o degradan hacia la región violada. Los límites

*) A. N. 44 I I p. 3 I 9 Z. 27 v. o. ist statt: $48^{\circ}$ I I' zu lesen: $48^{\circ}$. I I". Red. 
de las bandas por el lado del rojo están para el núcleo en $\lambda 5^{61 ;} 510$ y 472 y para la cola en $\lambda 55^{\circ}, 505$ y $46 \mathrm{I}$. Las observaciones son del dia 9 y del I I de mayo. La raya del sodio no se ha presentado visible, aunque se ha tratado de observarla con especial interés.

Adjunto, Fig. 4 en la lámina, envio un dibujo que representa el espectro tal como aparece en nuestro espectroscopio.

Observatorio de Madrid, i 6 de mayo i 9 Io. F. Iñiguez.

Nota: Las pruebas positivas, que remito, no reproducen todos los detalles delicados de las negatívas.

Während meiner Reise in Süd-Rußland gelang es mir viermal den Halleyschen Kometen zu beobachten:

I 9 10 April 27, Nicolajew, I 5\% ${ }^{\text {h }}$ Petersb. Zeit. Kern 3. Größe, Schweif kaum sichtbar.

Mai r, Nicolajew. Der Komet trat aus den Wolken um $15^{\mathrm{h}} 20^{\mathrm{m}}$. Scharf begrenzter Kern (für das unbewaffnete Auge); Helligkeit $2^{\mathrm{m}} \cdot 3$, Länge des Schweifs $\mathbf{I}^{1 / 2}{ }^{\circ}$. Im Fernrohr auf der der Sonne zugewandten Seite des Kerns ein haibkreisförmiger diffuser Schein sichtbar, Kondensation in dem nördlichen Teile desselben.

Mai 6 , Alupka, Krim, $\mathrm{x} 5^{\mathrm{h}}$. Kern scharf begrenzt, $2^{\mathrm{m}} \cdot 5$, gerader hellgelber Schweif von $14^{\circ}$ Länge, Richtung fast parallel mit $\omega, \iota, \gamma$ Piscium. Koordinaten des Schweifendes $\alpha=345^{\circ} \cdot 2, \delta=+4: 8$.

Mai 7, Jalta, Krim, I $5^{\mathrm{h}}$. Gleiche Helligkeit des Kerns mit $\alpha$ Andromedae $\left(2^{m} \cdot 1\right)$. Schweif länger und intensiver als am 6. Mai $\left(19^{\circ}-20^{\circ}\right)$. Koordinaten des Schweifendes $\alpha=343^{\circ} \circ, \delta=+5.8$.

In Jurjew war der Komet am östlichen Himmel überhaupt nicht zu sehen, am westlichen Himmel wurde er zum erstenmal am 24. Mai I I $^{\text {h }}$ Petersb. Zeit aufgefunden als lichtschwaches verwaschenes Objekt auf hellem Grunde ohne jegliche Andeutung eines Schweifes.

$$
\text { Jurjew (Dorpat), r9 1о Mai } 26 . \quad K \text {. Pokrowsky. }
$$

All' Osservatorio di Capodimonte, nelle mattine del 18 , I 9 e 20 maggio da $0^{\mathrm{h}}$ a $6^{\mathrm{h}}$ T. M. E., furono fatte ogni 6 minuti 20 letture della scala del magnete di declinazione: Je 60 medie ottenute in ciascun giorno non presentano alcun che di notevole, sia tra loro, sia da un giorno all'altro. In tutta la notte del 19 il cielo fu sereno-vaporoso: non si videro stelle cadenti, nè altri fenomeni notevoli; la luna tramontà a $2^{\mathrm{h}} 4 \mathrm{I}^{\mathrm{m}}$ T. M. E.; poco dopo, da $2^{\mathrm{h}} 45^{\mathrm{m}}$ a $3^{\mathrm{h}} \mathrm{I}^{\mathrm{m}}$ circa, si rese visibile all'oriente la coda della cometa; obliqua come nei giorni precedenti, in forma di triangolo, con un angolo molto acuto in $\theta$ Aquilae; il lato superiore passante per $\alpha$ Pegasi, che quasi restava fuori del chiarore della coda; il lato inferiore, passante al disotto di $\gamma$ Pegasi, che restava immersa nella coda a circa $1 / 10$ della sua larghezza in quel punto; ed il terzo lato, intersezione della coda coi vapori dell'orizzonte a circa $4^{\circ}$ di altezza, era lungo circa $6^{\circ}$. La luminosità decresceva dalla base al vertice opposto, in $\theta$ Aquilae, dove non è certo che giungesse.

La sera del ig il cielo fu coperto.

La mattina del 20 , nello stesso intervallo di tempo, da $2^{\mathrm{h}} 45^{\mathrm{m}}$ a $3^{\mathrm{h}} \mathrm{I} 5^{\mathrm{m}}$, con la luna non ancora tramontata, si vide all'oriente un chiarore più incerto nel contorno, più largo e meno lungo di quello veduto il giorno precedente: il lato inferiore passava tra $\alpha$ e $\gamma$ Pegasi, diretto ad $\alpha$ Aquilae; il lato superiore, pure diretto ad $\alpha$ Aquilae, passava al disotto. di $\delta$ Pegasi a circa $1 / 8$ della distanza tra $\delta$ Pegasi ed $\alpha$ Pegasi; ed il terzo lato era fatto dalla intersezione coi vapori dell'orizzonte. La luminosità, più intensa in basso, s'indeboliva gradatamente e svaniva prima di giungere al Delfino.

La mattina del 2 I fu nuvolosa, ed in quella del 22 : non si distinse alcun chiarore.

R. Osservatorio Astronomico di Capodimonte, I 9 10 Giugno 2.

F. Contarino.

Zur Zeit des Durchganges der Erde durch den Schweif des Halleyschen Kometen wurde in Kalocsa der Himmel unter günstigen Umständen mit Sorgfalt beobachtet: in der. Nacht des 18. Mai (astr.) und ebenso am I 9. Mai beständig. von Mitternacht bis zum Anbruch des Tages: Der Himmel war immer wolkenlos, anscheinend ganz klar; bei Tage sah man zerstreute Cirrus; die Sterne waren klar zu sehen; von irgend weichem ungewöhnlichen Lichtscheine war keine Spur zu bemerken; von Sternschnuppen wurden in der ersteren Nacht keine einzige gesehen, in der zweiten Nacht nur eine.

Ein Benndorfscher Registrierapparat für atmosphärische Elektrizität hat aber an beiden Tagen und Nächten ungewöhnlich lebhaft gearbeitet, wozu bei dem klaren, gleichmäßig. ruhigen Wetter und völliger Windstille in diesen Tagen kein Anlaß gegeben schien.

Kalocsa, I 9 10 Juni 6

F. Fénji, S. J.

\section{Bemerkung zu meiner Arbeit "Zur Statistik der spektroskopischen Doppelsterne" (A. N. 4415-16).}

Wie mir Herr Prof. Kobold mitteilt, legt Herr Doberck Wert auf den Hinweis, daß er bereits vor langer Zeit (A.N. $2168,3519)$ erkannt hat, daß bei visuellen Doppelsternsystemen die Exzentrizität mit der Periode zunimmt, wie ich dies für die spektroskopischen Doppelsterne in A. N. 44 I 5-1 6 nachweise. Ich hole diesen Hinweis auf die mir zur Zeit der Abfassung meiner Arbeit leider unbekannten Notizen
Dobercks gern nach, bemerke aber, daß ich zu meinen Untersuchungen durch die rein theoretischen Betrachtungen von See (Dissertation, Berlin I892) veranlaßt worden bin, der auch wohl zuerst in prägnanter Form auf die Verschiedenheit der Mittelwerte der Exzentrizitäten bei visuellen und spektroskopischen Doppelsternen aufmerksam gemacht hat (M. N, Vol. 68, p. 201 ). 